The Journal of Experimental Biology 204, 283-289 (2001)

Printed in Great Britain $₫$ The Company of Biologists Limited 2001

JEB2934

\title{
HYDROBIA ULVAE (GASTROPODA: PROSOBRANCHIA): A NEW MODEL FOR REGENERATION STUDIES
}

\author{
ALEXANDER M. GORBUSHIN ${ }^{1 *}$, IVAN A. LEVAKIN $^{1}$, NADEJDA A. PANCHINA ${ }^{2}$ AND YURI V. PANCHIN ${ }^{2,3}$ \\ ${ }^{1}$ Department of Histology and Cytology, Biology and Soil Sciences Faculty, St-Petersburg State University, Universitetskaya \\ nab. 7/9, St-Petersburg 199034, Russia, ${ }^{2}$ A. N. Belozersky Institute of Physico-Chemical Biology, Moscow State University, \\ Moscow, Russia and ${ }^{3}$ Institute of Problems of Information Transmission, Russian Academy of Sciences, Moscow, Russia \\ *e-mail: gorbushin@yahoo.com
}

Accepted 23 October 2000; published on WWW 3 January 2001

\begin{abstract}
Summary
Within 2 weeks of decapitation, Hydrobia ulvae was able to regenerate new head structures including buccal ganglia. It was also capable of regenerating propodial ganglia after anterior foot amputation. The functional regeneration of the buccal ganglia was demonstrated by behavioural observations and by electrophysiological experiments. The presence of the oesophagus was shown to

animals in future studies, the effects of age, sex and trematode infestation on the regeneration capacity of H. ulvae have been evaluated. The high capacity for regeneration together with the possibility of using electrophysiological techniques makes $H$.ulvae a favourable model in which to study neurogenesis in adult animals.
\end{abstract} be important for regeneration of the buccal complex. H. ulvae provides a new model for regeneration studies, so details of the topographic anatomy and biology of this species are described. To standardize experimental
Key words: age, electrophysiology, Hydrobia ulvae, morphogenesis, neurogenesis, regeneration, Trematoda, Cryptocotyle cancavum.

\section{Introduction}

The potential for regeneration is present to varying degrees throughout the animal kingdom. In terms of their capacity to regenerate, molluscs are probably best known for being able to replace various body parts: the eyes, tentacles, penis and siphon and regions of the foot, mantle and shell can all be regenerated to some degree by most molluscs (Hyman, 1967; Moffett, 1995). Gastropod molluscs have been good subjects for the investigation of regeneration following injury to the nervous system because of their relative simplicity. They grow new tentacles with associated tentacle ganglia, selectively reinnervate peripheral targets, repair central connections and may even replace lost neurons and ganglia (Moffett, 1995). Apart from the tentacle ganglia, however, a capacity to repair the ablated ganglia has been reported in only one snail species, Melampus (Price, 1977). In this animal, the ablated cerebral ganglion was replaced by a new structure in which the formation of some differentiated neurons was detected 5-6 months after operation. In a single long-term (12 months) regenerate animal, the cerebral ganglion was found to have regenerated with topographic features that were similar to, but slightly smaller than, those of the intact ganglion (Moffett and Austin, 1982). In this paper, we demonstrate that the marine prosobranch Hydrobia ulvae (Pennant) displays a remarkable regenerative capacity. Two weeks after decapitation, H. ulvae was able to regenerate different head structures including buccal ganglia. It was also capable of regenerating propodial ganglia after amputation of the anterior part of the foot (propodium). The functionality of the regenerated buccal ganglia was demonstrated both by behavioural observations and by electrophysiological experiments. Since H. ulvae may provide a useful model for regeneration studies, this paper describes the topographic anatomy and biology of this species.

Although keeping this animal under laboratory conditions is easy, the presence in its life cycle of a planktonic veliger stage makes breeding the snails under artificial conditions difficult. It is, therefore, necessary to collect experimental material from natural populations, which are quite different in sex ratio, age structure and parasite (Trematoda) infestation. The effects of these three factors on the regeneration capacity of Hydrobia ulvae have, therefore, been evaluated in the current study.

The fast rate and high reliability of head regeneration in H. ulvae makes it possible to investigate the development of head structures under different experimental conditions and to study the influences of one head structure on the regeneration of others. We show here that the buccal mass with associated ganglia successfully regenerates when excised from the animal without decapitation and that the presence of the gut (oesophagus) in the vicinity of the regeneration site is necessary for the normal development of head structures. 


\section{Materials and methods}

\section{Experimental animal}

Experiments were carried out at the White Sea Marine Biological Station, Kartesh. The experimental animal Hydrobia ulvae (Pennant), known as the mud snail, is one of two native representatives of the family Hydrobiidae in the White Sea. It typically inhabits mudflats and creeks in protected bays and estuaries. The species is spread throughout the northern shores of Western and Eastern Europe and the Atlantic shore of France. This small snail (up to $8 \mathrm{~mm}$ in shell height) has a maximum lifespan of 4 years in the White Sea. Sexes are separate in H. ulvae and males are phallic. Development is indirect and the veliger stage is planktotrophic. H. ulvae is a size-selective deposit-feeder consuming mainly benthic diatoms and the bacterial film covering organic aggregates.

Eight different trematode species use H. ulvae as an intermediate host in Sukhaja Salma. The most common parasite Cryptocotyle cancavum (Creplin) (Trematoda: Heterophyidae), which accounts for approximately $60 \%$ of all infections, has been used to determine whether parasitism influences regeneration processes in H.ulvae. Parthenogenetically generated $C$. cancavum develop as rediae within the gonads of the snails.

There is one previous study describing the morphology of H. ulvae (Krull, 1935), and it is important to note some details of the topographic anatomy of the head. The head of H. ulvae extends into a long snout that tapers slightly, expanding at the tip into two lobes. The mouth is situated between these lobes. The tentacles are long and slender, and each has an eye set on a lateral bulge at its base. The oral tube is very short and, situated on its inner side, lies the buccal cavity. This is a globular chamber leading to two posteriorly positioned tubes, the upper of which is the anterior oesophagus and the lower the radular sac. The roof of the buccal cavity is attached to the body wall by numerous strands of muscle, while the floor is raised into a tongue-like prominence known as the buccal mass or odontophore. The buccal mass is a very mobile structure, which can turn and twist in all directions; over the median part of its mid-dorsal surface, runs the radula. Two salivary glands lie dorsal to the oesophagus, and their ducts enter the buccal cavity. The oesophagus leads from the buccal cavity (in the head) to the stomach (in the visceral mass). At the posterior end of the anterior oesophagus, the gut is surrounded by a nerve ring, beyond which is the midoesophagus, which extends to the posterior end of the mantle cavity, where it joins the posterior oesophagus. The nervous system of $H$. ulvae consists of seven paired and two unpaired ganglia (Figs 1, 2A). A noteworthy feature of its anatomy is the association of two pairs of ganglia with the pedal ganglion: the propodial ganglia and metapodial ganglia. The propodial ganglia innervate the anterior part of foot and the metapodial ganglia the posterior foot. Buccal ganglia are positioned at the point where the anterior oesophagus and the radular sac separate from the buccal cavity.

\section{Collection of specimens and maintenance in the laboratory}

Snails were collected at the beginning of August 1999 from mudflats exposed at low tide in the inner part of Sukhaja Salma creek (Chupa inlet, Kandalaksha bay of the White Sea). The molluscs were brought to the laboratory and kept in 31 storage tanks filled with water at a salinity of $24 \% \mathrm{o}$. The temperature of the tanks was maintained at $10^{\circ} \mathrm{C}$. The animals were supplied ad libitum with food, the filamentous alga, Enteromorpha spp., ground to powder. Food remains were removed and the sea water was changed daily.

The snails were separated into infected individuals (shedding C. cancavum cercariae) and uninfected specimens (not shedding cercariae), identified as males and females according to the presence or absence of a penis and their age, estimated ( 2 and 3 years old) according to the number of winter-grade lines on the shell and to their size in relation to individuals in corresponding age groups. To determine whether

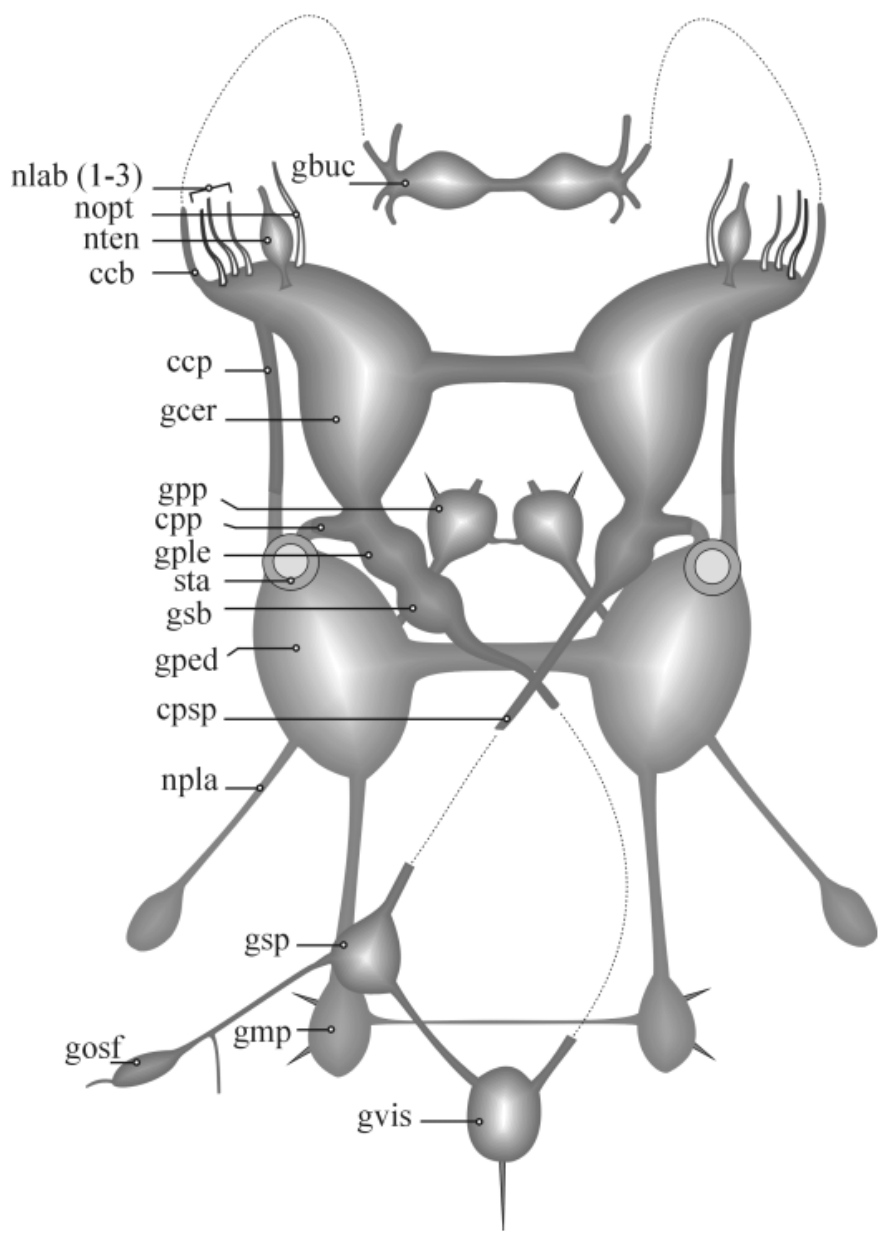

Fig. 1. A drawing of the nervous system of Hydrobia ulvae. ccb, cerebrobuccal connective; ccp, cerebropedal connective; cpp, pleuropedal connective; cpsp, pedal supraoesophageal connective; gbuc, buccal ganglion; gcer, cerebral ganglion; gmp, metapodial ganglion; gosf, osphradial ganglion; gped, pedal ganglion; gple, pleural ganglion; gpp, propodial ganglion; gsb, sub-oesophageal ganglion; gsp, supra-oesophageal ganglion; gvis, visceral ganglion; nlab, nervus labialis; nopt, nervus opticus; nten, nervus tentacularis; npla, nervus pedolateralis; sta, statocyst. 
an individual that was not shedding cercariae was really uninfected (the absence of shedding does not necessarily indicate that a snail is uninfected, since mature cercariae may not be present), all snails from the 'uninfected' group were dissected after experiments, and their soft parts were examined under a dissection microscope for the presence of parasites.

\section{Surgical operations}

All surgery was performed on ice in cold filtered sea water to anaesthetize and achieve better muscle relaxation. Two types of decapitation were performed ('decapitation' means removing the buccal mass and associated ganglia but not the cerebral ganglia). Type I involved excision of the anterior part of head with the radular sac, buccal ganglia, tentacles, eyes and the anterior part of foot (propodium) together with the propodial ganglia (Fig. 2B). This resulted in transection of the oesophagus, the salivary gland ducts, the cerebrobuccal connectives, the nervus tentacularis, $n$. opticus and three pairs of $\mathrm{n}$. labialis as well as pedal-propodial connectives. Type II decapitation is similar to type I, but the propodium and
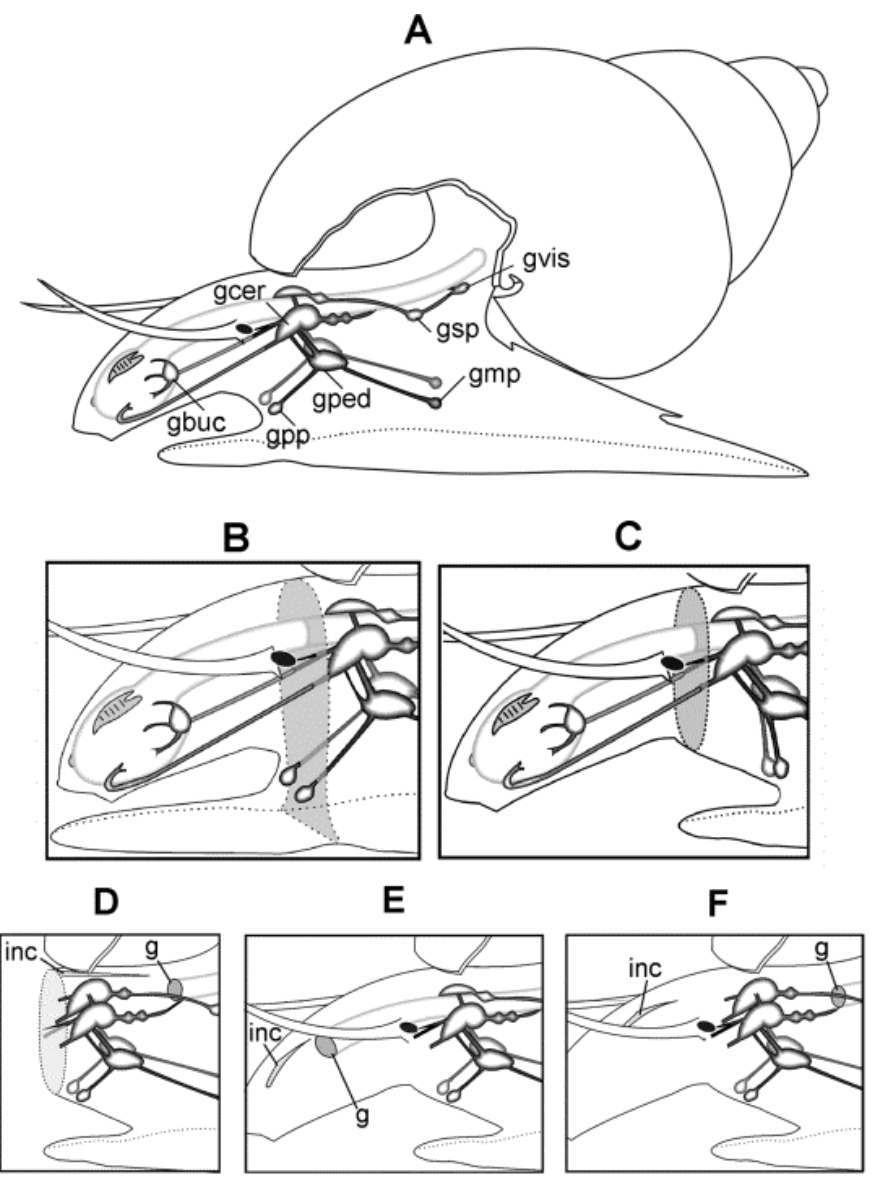

Fig. 2. Topographic anatomy of the intact Hydrobia ulvae nervous system (A) and following type I (B) and type II (C) decapitation. Additional surgical operations are shown in D-F. (D) Type II decapitation with a portion of the gut ( $\mathrm{g}$ ) removed; (E) buccal complex removed through the small incision (inc) without decapitation; (F) buccal complex with a portion of the gut removed, For further abbreviations, see Fig. 1. propodial ganglia were not removed (Fig. 2C). After decapitation, the removed parts were screened carefully to ensure the quality of the operation. No suturing or antibiotics were required.

Preliminary results indicated that the following characteristics were relevant stages of regeneration that could be used to estimate regeneration rates in groups of H. ulvae of different quality: (i) the appearance of black eye pigment, (ii) the formation of tentacle buds and (iii) the restoration of the ability to feed (monitored by faecal pellets production). In the decapitation experiment, 95 specimens (see Table 1) were type-I-operated and were kept at $10^{\circ} \mathrm{C}$ after operation. Monitoring of regeneration events was performed daily under a dissection microscope (magnification $\times 32$ ). Data analysis was performed using an exact Fisher test and a two-tailed ttest.

In addition, photographic records were made using 10 uninfected 2-year-old females subjected to a type I operation and subsequently kept at $20^{\circ} \mathrm{C}$. In five animals, decapitation was carried out as a type II operation, but included removal of the gut (oesophagus) from the site of regeneration. To do this, an additional excision was made several millimetres posterior to decapitation cut, and a portion of the gut was pulled out and removed (Fig. 2D). In 11 experiments, the buccal complex and associated ganglia were removed through a small incision in the head without decapitation of the animals (Fig. 2E). In four of these animals, a portion of the gut was removed as described above (Fig. 2F). All animals were kept in filtered sea water for 30 days before dissection to study the regeneration of head structures.

\section{Electrophysiology}

For electrophysiological experiments, buccal ganglia were dissected from intact or regenerating animals and treated for 2 $\min$ in $0.5 \%$ Pronase $\mathrm{E}$ in sea water to soften the sheath. The ganglia were then rinsed in a large volume of sterile sea water. The preparations were placed into $35 \mathrm{~mm}$ polystyrene dishes (Falcon 3801) lined with agarose gel prepared with sea water and held in place with a thin layer of thick agarose gel. Intracellular recordings were carried out with glass

Table 1. The proportion of animals showing successful regeneration of tentacles and eyes and the ability to feed in different snail groups

\begin{tabular}{|c|c|c|c|c|c|}
\hline \multirow{2}{*}{ Snail group } & & \multicolumn{3}{|c|}{ Success $(\%)$} & \multirow{2}{*}{$\begin{array}{c}\text { Sample } \\
\text { size }\end{array}$} \\
\hline & & Tentacles & Eyes & Feeding & \\
\hline \multirow[t]{2}{*}{ Age (years) } & 2 & 91 & 83 & 94 & 47 \\
\hline & 3 & 88 & 83 & 79 & 48 \\
\hline \multirow{2}{*}{$\begin{array}{l}\text { Trematode } \\
\text { infection }\end{array}$} & Infected & 93 & 85 & 85 & 41 \\
\hline & Uninfected & 87 & 81 & 87 & 54 \\
\hline \multirow[t]{3}{*}{ Sex } & Male & 82 & 86 & 68 & 48 \\
\hline & Female & 92 & 77 & 81 & 47 \\
\hline & Total & 89 & 83 & 86 & 95 \\
\hline
\end{tabular}


microelectrodes filled with $3 \mathrm{~mol} \mathrm{l}^{-1} \mathrm{KCI}$ and with tip resistances of 50-100 M $\Omega$. All chemicals were from Sigma.

\section{Results}

\section{Morphology of regeneration}

Some of the stages of regeneration following decapitation are shown in Fig. 3A-D. In both types of decapitation experiment, regeneration followed a similar overall course. Immediately after decapitation, snails withdrew into their shells, but they emerged within 1-2 h, enabling the area of the wound to be observed. In most cases, body wall muscle contraction caused the wound to close within a few minutes. The initial stage of recovery was the formation of a flat epithelial layer over the surface of the wound within I day of decapitation. This then swelled and formed lateral bulges (Fig. 3A), which became the loci of both tentacle and eye bud differentiation. Regenerating tentacles formed as small stickshaped buds, while eyes appeared as invaginations on the top of small bulges. The formation of the tentacle and eye buds coincided with the differentiation and intensive growth of the proboscis (Fig. 3B). After type I operations, a bud representing the regenerating propodium formed in the ventral region of the scar. The next stage of recovery was the appearance of black pigment in the eye invaginations. This was followed by the initiation of locomotion in the growing propodium, which exhibited normal coordination in all cases following a type I operation (Fig. 3C). At the same time, it was possible to distinguish the developing buccal complex, which appeared as round unpigmented bodies through the newly formed

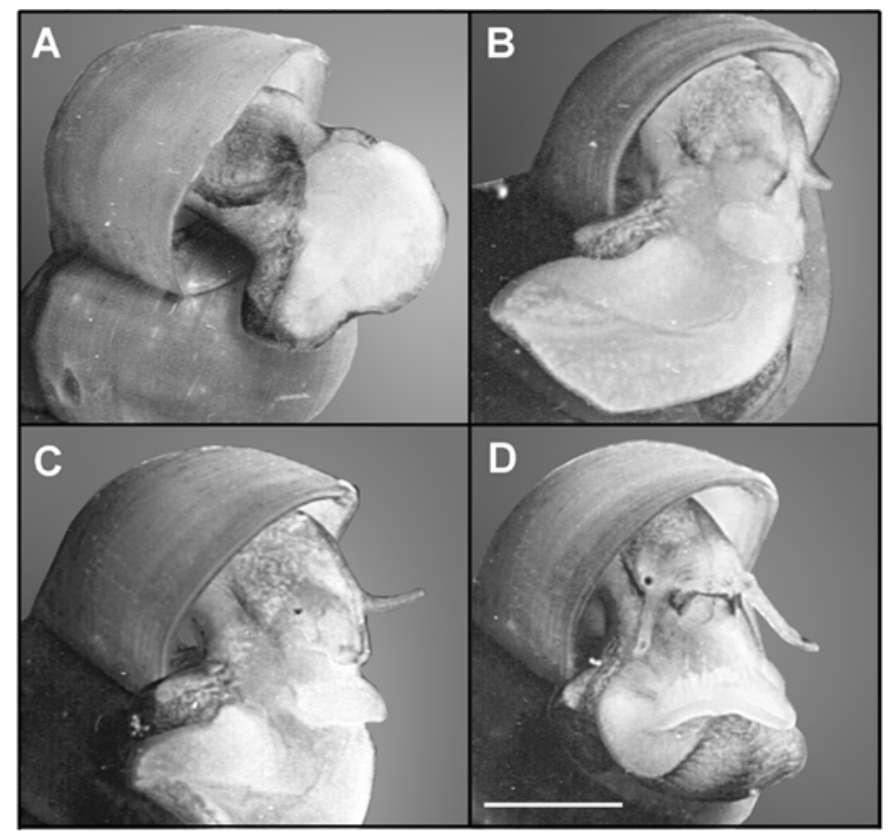

Fig. 3. Regeneration in Hydrobia ulvae after decapitation (type I, $20^{\circ} \mathrm{C}$ ) at 3 days (A), 5 days (B), 8 days (C) and 15 days (D) postoperation. For a detailed account of recovery, see the text. Scale bar $1 \mathrm{~mm}$.

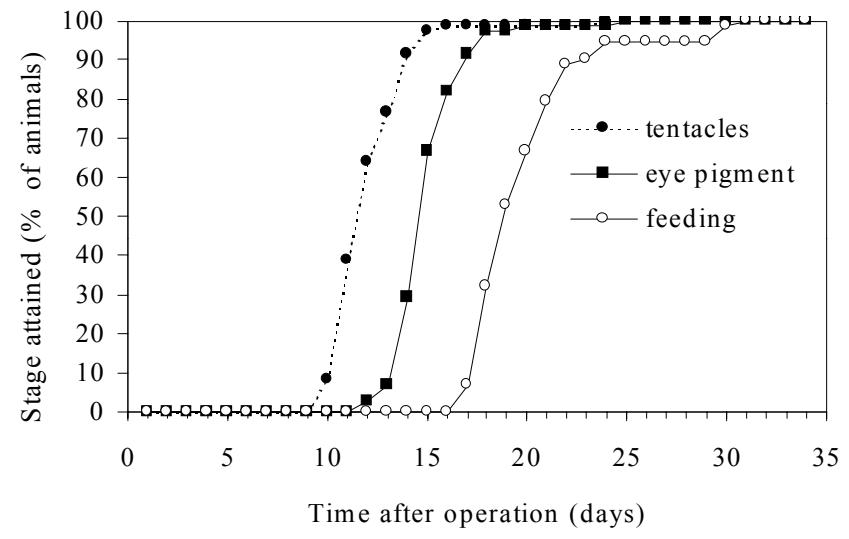

Fig. 4. Regeneration in Hydrobia ulvae after decapitation. The stages of repair were categorized as tentacle bud formation, eye pigment appearance and faecal pellet formation (initiation of feeding).

transparent body wall. Relatively large buccal ganglia could easily be found at this stage in dissected specimens. Later, the skin lost its transparency and gradually became pigmented; the snails eventually began feeding and producing faecal pellets. At this stage, morphogenetic processes were complete and the head of a successfully regenerated snails appeared to have all the features of a normal snail, albeit the head was smaller in size and lighter in pigmentation. Subsequently, the head organs continued to show uniform growth (Fig. 3D).

Twenty-six out of 95 specimens $(27 \%)$ showed detectable morphogenetic anomalies after decapitation. Snails with an abnormally developed mouth and/or asymmetrically developed eyes and tentacles were judged unsuccessful in regeneration (Table 1). There was no significant effect $(P>0.05)$ of the sex of the snail, its age or the presence of infection with trematodes on the successful regeneration of tentacles and eyes. Similarly, 'sex' and 'infection' had no effect on the recovery of feeding behaviour. However, the proportion of snails that began feeding showed an insignificant $(\mathrm{P}=0.07)$ tendency to be higher in younger individuals than in older ones.

Despite the fact that type I decapitation was highly traumatic, regeneration in H. ulvae was rapid: recovery of feeding ability was apparent by the seventeenth day after the operation. The time course of regeneration is shown in Figs 4 and 5. When the possible effects of infestation with trematodes on regeneration rate and the effects of the sex and age of the snails were examined, it was found that parasitism and age had no effect on morphogenetic stages such as tentacle bud and eye pigment formation ( $\mathrm{P}>0.05$; Fig. 6A,B). However, when sex was considered as a factor, it was shown to have a significant effect: males formed tentacle buds and eye pigment significantly faster $(\mathrm{P}=0.02$ for tentacle buds and $\mathrm{P}=0.03$ for eye pigment) than females (Fig. 6C).

\section{Functional recovery}

During the first 6-12 $\mathrm{h}$ after operation, regenerating snails showed a tendency to be hypersensitive to mechanical stimulation. Thus, for instance, they responded to slight 
vibration with body withdrawal, whereas intact snails made no response to such a stimulus. However, this hypersensitivity disappeared over time. Normal responses were restored coincident with the period of eye pigment formation and/or the recovery of tentacle sensitivity. Tentacle retraction reappeared when the organ grew to approximately half its normal length. To attach to the substratum, H. ulvae uses the propodial part of the foot controlled by the propodial ganglia. After type I decapitation (ablation of the propodium together with the propodial ganglia), snails temporarily lost normal locomotion, but regained this within approximately 15 days. After type II decapitation, in which these ganglia were left intact, snails attempted to crawl within approximately I h of the operation. The potential for complete functional recovery after severe lesions shown by $H$. ulvae was most evident in the restoration of feeding ability. The mouth musculature initiated contractions within 1-2 days of mouth formation. This was followed by movements of the regenerating buccal complex, visible through the body wall, although the snails were not yet able to feed. In the first decapitation experiment, rhythmic retractions of the radular sac, controlled by the regenerated buccal ganglia, were seen 14-19 days postoperation. Approximately, 21 days after decapitation in the first experiment, the regenerating snails began to produce faecal pellets. Eventually, 82 out of 95 individuals (86\%) recovered their ability to feed and showed normal locomotion and feeding behaviour. When successfully regenerated snails were observed for several months, no distinct morphological or behavioural postoperative abnormalities were found.

The rate of recovery of normal feeding was significantly affected by all three factors studied. Uninfected snails began to ingest food earlier than infected ones ( $\mathrm{P}=0.02$; Fig. 6A), younger snails earlier than older snails $(\mathrm{P}=0.02$; Fig. $6 \mathrm{~B})$, and males earlier than females $(\mathrm{P}=0.04$; Fig.6C).

\section{Electrophysiology}

In most gastropods (Cohen et al., 1978; Benjamin and Rose, 1979; Kater, 1974; Arshavsky et al., 1988; Arshavsky et

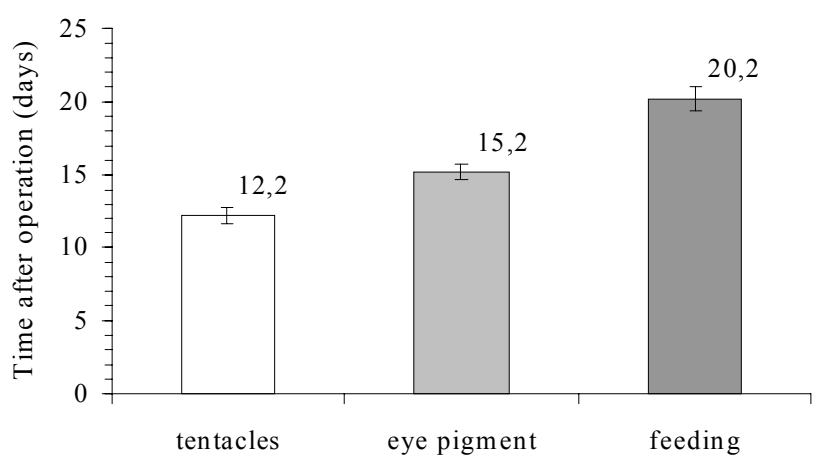

Fig. 5. Rate of regeneration of Hydrobia ulvae after decapitation (values are means $\pm 95 \%$ confidence interval). The three categories are first appearance of tentacle buds, appearance of eye pigment and faecal pellet formation. $N$, see Table 1 .

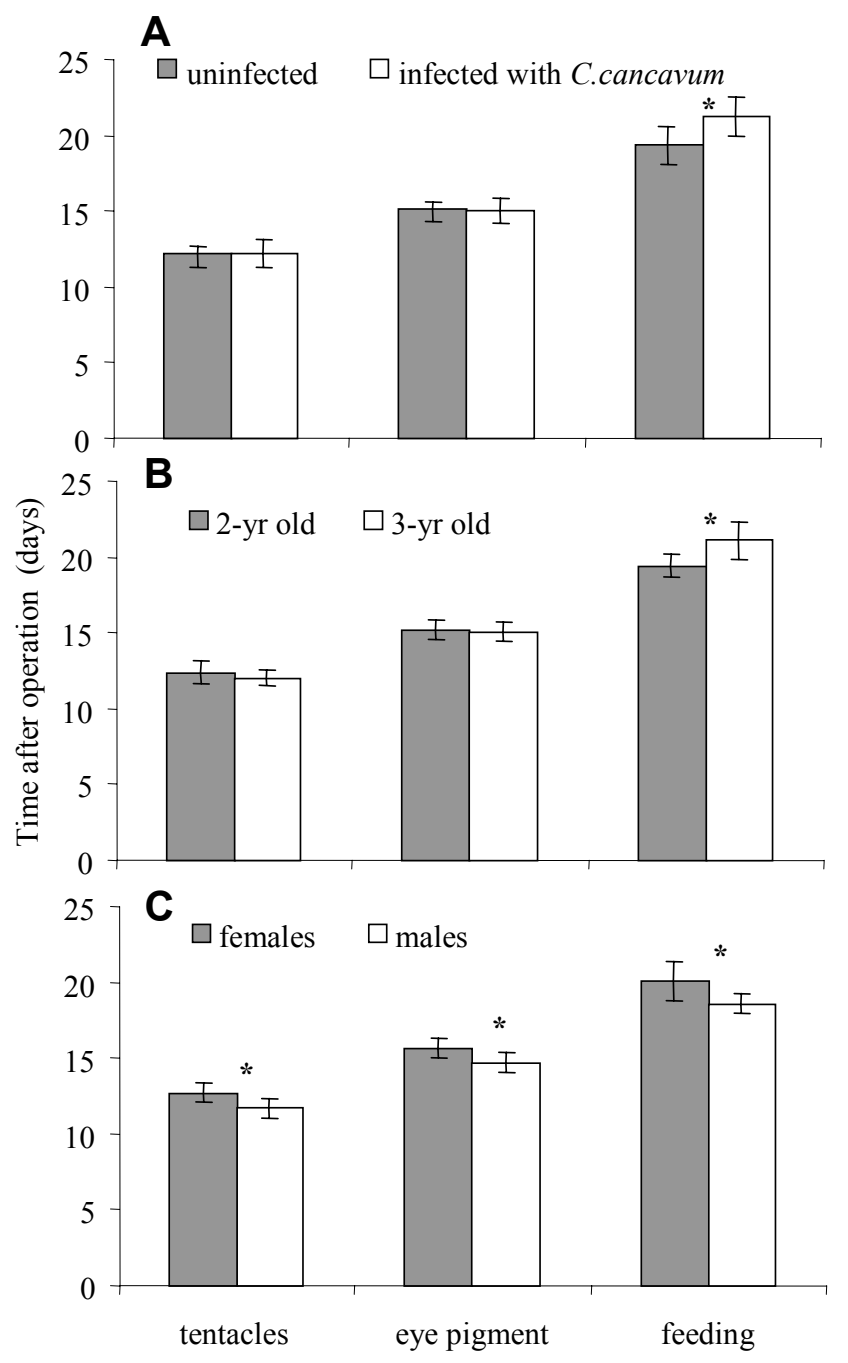

Fig. 6. Regeneration rate in Hydrobia ulvae of different groups after decapitation (values are means $\pm 95 \%$ confidence interval). An asterisk indicates a significant difference $(P<0.05)$ between groups. Categories are as in Fig. $4 ; N$ values are given in Table 1.

al., 1989), including prosobranchs (Arshavsky et al,, 1983; Furukawa and Kobayashi, 1985), the neuronal activity that controls feeding movements persists in isolated buccal ganglia (fictive feeding). This activity is driven by the central pattern generators (CPGs) and can be used to demonstrate the functional recovery of the buccal ganglia neuronal circuit. All the randomly recorded unidentified neurons $(\mathrm{N}=7)$ recorded in isolated buccal ganglia of untreated animals displayed similar and uniform bursting activity (Fig. 7A) with a frequency related to normal feeding movements (1.5-4 cycles s $\left.\mathrm{s}^{-1}\right)$. Essentially the same pattern of activity was recorded from isolated regenerated ganglia (after 10 days of recovery, type-Ioperated animals, $N=5$ ) in operated animals (Fig. 7B).

\section{Buccal complex regeneration}

Following decapitation, the buccal complex develops as an integrated part of a completely new regenerating head. Can this 


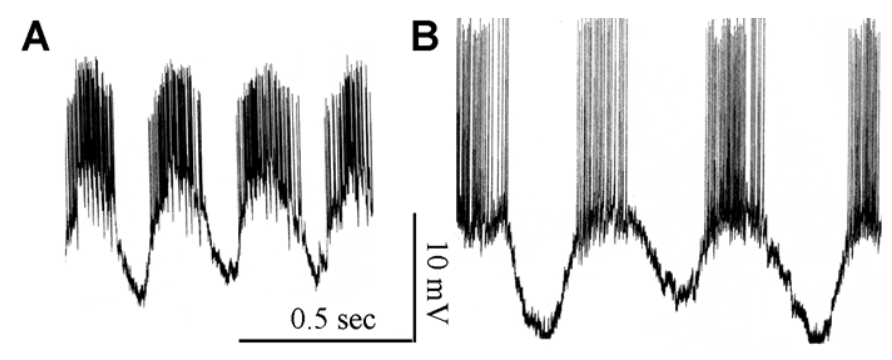

Fig. 7. Examples of intracellular recordings of the feeding rhythm from the buccal neurons in control (A) and regenerating (B) (10 days after decapitation) buccal ganglia.

structure regenerate by itself? To study this question, the buccal complex was removed from the animal with only minor injures to other head structures. In seven animals, the buccal complex (including the buccal ganglia) was successfully extirpated through a small incision in the dorsal head skin above the complex. Thirty days after surgery, an apparently normal regenerated buccal complex was found in all these animals. It was connected to the initial mouth on one side, to the oesophagus on the other and possessed all the associated substructures such as odontophore, radula, salivary glands and buccal ganglia. The appearance of the regenerated buccal complex in these experiments was the same as in decapitated animals 20-30 days post-surgery.

\section{The role of the oesophagus in the regeneration of head structures}

One of the main functions of the head structures is to transfer food through the mouth and buccal complex to the oesophagus. How, then, would the head structures develop if the oesophagus were removed from the head cavity? In five animals, decapitation similar to the type I operation was accompanied by a removal of the gut (oesophagus) from the site of regeneration via an incision in the skin several millimetres posterior to the cut (Fig. 2). In these animals, the wound closed and in three cases the tentacles and eyes had differentiated by the time the animals were dissected (30 days). However, no mouth formed, and dissection revealed that the buccal complex was absent. A blind-ending oesophagus was located outside the head cavity in the region of the foot stalk. No signs of ectopic regenerating head structures were detected in this area. Similar results were obtained when the oesophagus was removed from the site of regeneration in experiments in which the buccal complex was extirpated. In all cases of this kind $(\mathrm{N}=4)$, the buccal complex failed to regenerate and the head cavity was empty.

\section{Discussion}

Although molluscs are known to have considerable powers of regeneration, the phenomenon described in this paper is probably the most striking example of structural and functional recovery in a snail after removal of major body parts. The gastropod mollusc Hydrobia ulvae can regenerate its head or some head structures and part of the foot with associated ganglia in less than 2 weeks. Such a degree of regeneration is more usually associated with turbelarian and polychaete worms than with molluscs. What underlies this high regenerative capacity in H. ulvae? In general, animals inhabiting the intertidal zone must develop an ability to resist pressure from unfavourable environmental factors. Unpredictable and wide-ranging changes in temperature, salinity and moisture put pressure on mud snails to develop highly effective mechanisms of cellular and tissue repair. In addition, a capacity for organ regeneration is likely to have developed as a way of resisting predation. Because of its high population density, H. ulvae forms a common prey for intertidal polychaetes, crustaceans and sea bird, so that recovery from loss of body parts would give the snails a considerable advantage over animals with poor regenerative capabilities. It was the discovery of a decapitated specimen during field sampling by A. Gorbushin (unpublished observations) several years ago that suggested the adaptive nature of this unique regeneration ability in H. ulvae and provoked this study.

Regeneration in H. ulvae is reliable and uniform. The level of successful recovery does not depend on the sex or age or even the level of parasite infection of the animal. This last finding (which may seem rather unusual since parasites are usually regarded as harmful to their hosts) does not contradict the fact that parasitized H. ulvae show increased somatic growth (gigantism) (Rothschild and Rothschild, 1939; Gorbushin, 1997). Our failure to demonstrate a high proportion of morphogenetic abnormalities in regenerating $H$. ulvae infected with C. cancavum suggests that the interaction between host and parasite is not highly antagonistic. However, parasitism did slow down regeneration to some extent (by approximately 1.5 days), an effect similar to that seen with increasing age. Females also showed a similar slowing of regeneration processes, and the fact that gender may influence the rate of functional recovery is quite intriguing. It is possible that investment of energy into repair (as part of normal maintenance processes) is lower in females because the energy expended in egg production is likely to be higher than the energy expended in the production of spermatozoids in males. In other words, females sacrifice effectiveness of repair processes for the sake of reproduction to a greater degree than do males.

Even though prosobranch molluscs are less favourable for electrophysiological study than opisthobranchs and pulmonates, the neurons from $H$. ulvae ganglia are suitable for intracellular recordings. The possibility of combining the advantage of rapid regeneration with an electrophysiological approach makes H. ulvae an attractive model for neurogenesis studies. Our electrophysiological data demonstrate that neurons in the regenerated buccal ganglia are active and produce patterns of rhythmic activity similar to those of intact animals. This indicates that the buccal neuronal network is restored, and that buccal movements are controlled by this network rather than by innervation from undamaged nonbuccal ganglia. Indeed, a regenerated buccal mass with attached buccal ganglia continues to perform rhythmic feeding movements when isolated from the animal. A fundamental problem when studying the regeneration of complex structures 
is to understand how the regenerating system determines which parts are missing after amputation and how it ensures the correct arrangement of different regenerating structures both with each other and with pre-existing undamaged structures without duplication or loss of essential parts. When the head of $H$. ulvae re-forms after decapitation, a number of separate events have to be coordinated to achieve functional regeneration. The odontophore and radula are produced, a mouth is formed, the gut is attached to the buccal mass and buccal ganglia develop to provide correct innervation.

One way of dissecting the different events in head regeneration that may lead to a better understanding of the mechanisms involved is to remove individual parts of the head complex and to study whether these can regenerate by themselves and how each structure affects the regeneration of other structures. H. ulvae can act as a model providing a broad spectrum of such preparations. In this paper, we have demonstrated that the buccal complex can regenerate autonomously. This indicates that regeneration is a complex program that is not necessarily executed as a single event (regeneration of the head), but that the animal can differentiate which parts are missing and can restore them separately. This task may be even more complex than de novo formation of the whole head because it requires the formation of correct connections between the new structure and existing systems, so that, for example, the buccal complex has to be connected to the mouth and to the oesophagus.

The presence of the oesophagus in the vicinity of the regeneration site seems to be important for the formation of the buccal complex both in decapitated animals and after buccal complex extirpation. However, the presence of a segment of oesophagus does not induce the formation of an ectopic buccal complex in a novel position. The absence of the oesophagus does not prevent formation of other head structures such as the eyes and the tentacles. Further experiments are necessary to understand which tissues play inductive roles and which contribute new cells for the regeneration of the head structures.

The unique capacity of H. ulvae for extensive regeneration, together with the possibility of monitoring nerve activity using an electrophysiological approach, makes this a suitable model for the study of neurogenesis in adult animal, which will complement the more usual embryological approach and may provide new insights into our understanding of the formation of the nervous system.
This investigation was supported by the Russian Foundation of Basic Research (RFBR) grant N 99 04-48992, 99 04-63080, 00 04-49434 and by the program 'Universities of Russia', grant N 3918.

\section{References}

Arshavsky, Yu. I., Beloozerova, I. N., Orlovskii, G. N. and Panchin, Yu. V. (1983). Effect of serotonin and theophylline on generation of rhythmic activity in the buccal ganglia of gastropod mollusks. Neirofiziologiia 15, 321-323 (in Russian).

Arshavsky, Yu. I., Deliagina, T. G., Meizerov, E. S., Orlovsky, G. N. and Panchin, Yu. V. (1988). Control of feeding movements in the freshwater snail Planorbis comeus. 1. Rhythmical neurons of buccal ganglia. Exp. Brain Res. 70, 310-322.

Arshavsky, Yu. I., Deliagina, T. G., Orlovsky, G. N. and Panchin, Yu. V. (1989). Control of feeding movements in the pteropod mollusc, Clione limacina. Exp. Brain Res. 78, 387-397.

Benjamin, P. R. and Rose, R. M. (1979). Central generation of bursting in the feeding system of the snail, Lymnaea stagnalis. $J$. Exp. Biol. 80, 93-118.

Cohen, J. L., Weiss, K. R. and Kupferman, I. (1978). Motor control of buccal muscles in Aplysia. J. Neurophysiol. 41, 157180.

Furukawa, M. and Kobayashi, M. (1985). Neural mechanisms underlying the feeding movements of a mollusc Rapana thomasiana. Comp. Biochem. Physiol. 81A, 779-786.

Gorbushin, A. M. (1997). Field evidence of trematode-induced gigantism in Hydrobia spp. (Gastropoda: Prosobranchia). J. Mar. Biol. Ass. U.K. 77, 785-800.

Hyman, L. H. (1967). The Invertebrates. vol. 6, Mollusca I. New York: McGraw-Hill.

Kater, L. (1974). Feeding in Helisoma trivolvis: the morphological and physiological bases of a fixed action pattern. Am. Zool. 14, 1017-1036.

Krull, H. (1935). Anatomische Ultersuchungen an einheimischen Prosobranchiem und Beintrage zur Philogenie der Gastropoden. Zool. Jb. Abt. Anat. 60, 399-64.

Moffett, S. B. (1995). Neural regeneration in gastropod molluscs. Prog. Neurobiol. 46, 289-330.

Moffett, S. B. and Austin, D. R. (1982). Generation of new cerebral ganglion neurons in the snail Melampus: an ultrastuctural study. J. Comp. Neurol. 207, 177-182.

Price, C. H. (1977). Regeneration in the central nervous system of a pulmonate mollusc Melampus. Cell Tissue Res. 180, 529-536.

Rothschild, A. and Rothschild, M. (1939). Some observations on the growth of Peringia ulvae (Pennant 1777) in the laboratory. Nov. Zool. 41, 240-247. 